\title{
BUSINESS PROCESS SIMULATION FOR CLAIMS TRANSFORMATION
}

\author{
Mark Grabau \\ Advanced Analytics and Optimization \\ IBM Corporation \\ Westerville, OH 43082, USA
}

\author{
Quinn Conley \\ Melissa Marshall \\ Analytics Resource Center \\ Westfield Insurance \\ Westfield Center, OH 44251, USA
}

\begin{abstract}
Westfield Insurance is undertaking a replacement of its legacy claims system. They had several business process and staffing changes they were considering; however, they had no way to test their options prior to implementation. After successfully simulating the First Notification of Loss (FNOL) to adjuster assignment process, Westfield decided to append the rest of the claims process. This resulted in an end-toend claims process simulation, including adjudication, settlement, litigation, salvage, subrogation claims and fraud. The process, results, and lessons learned are discussed.
\end{abstract}

\section{INTRODUCTION}

Westfield Insurance is undertaking a replacement of its legacy, mainframe claims system. Westfield was considering several business process and organizational changes in conjunction with the replacement. With months of lead time before the start of systems development, Westfield decided to simulate the front end of the claims process from the time the claimant or agent notifies Westfield of the incident until the claim is correctly assigned to an adjuster. Upon completion of that project, Westfield chose to model the entire claims process end-to-end including adjudication, settlement, litigation, salvage, subrogation, and fraud. This paper describes the process that was modeled in detail, how atypical processes were modeled, and the results of the project.

\section{THE PROCESS}

Insurance claims processing is extremely complex. Claims arrive through different channels, with each channel involving different processes and resources to assign the claim to the correct adjuster. There are various types of resources that can touch each claim and each type may vary in skill level, experience, and tenure. Adjusters will typically have dozens of claims in process at any given time, with some claims unsettled for months or even years. A high-level process diagram can be seen in Figure 1. Each step in the process will be briefly described.

\subsection{First Notification of Loss}

Claims are often submitted to insurers through multiple channels including fax, email, or phone. Claimants and insureds can submit claims directly to Westfield, or if the insured is submitting the claim, they may do so through their independent agent. The information is often received in the form of a fax, which must then be entered into the claims system. 


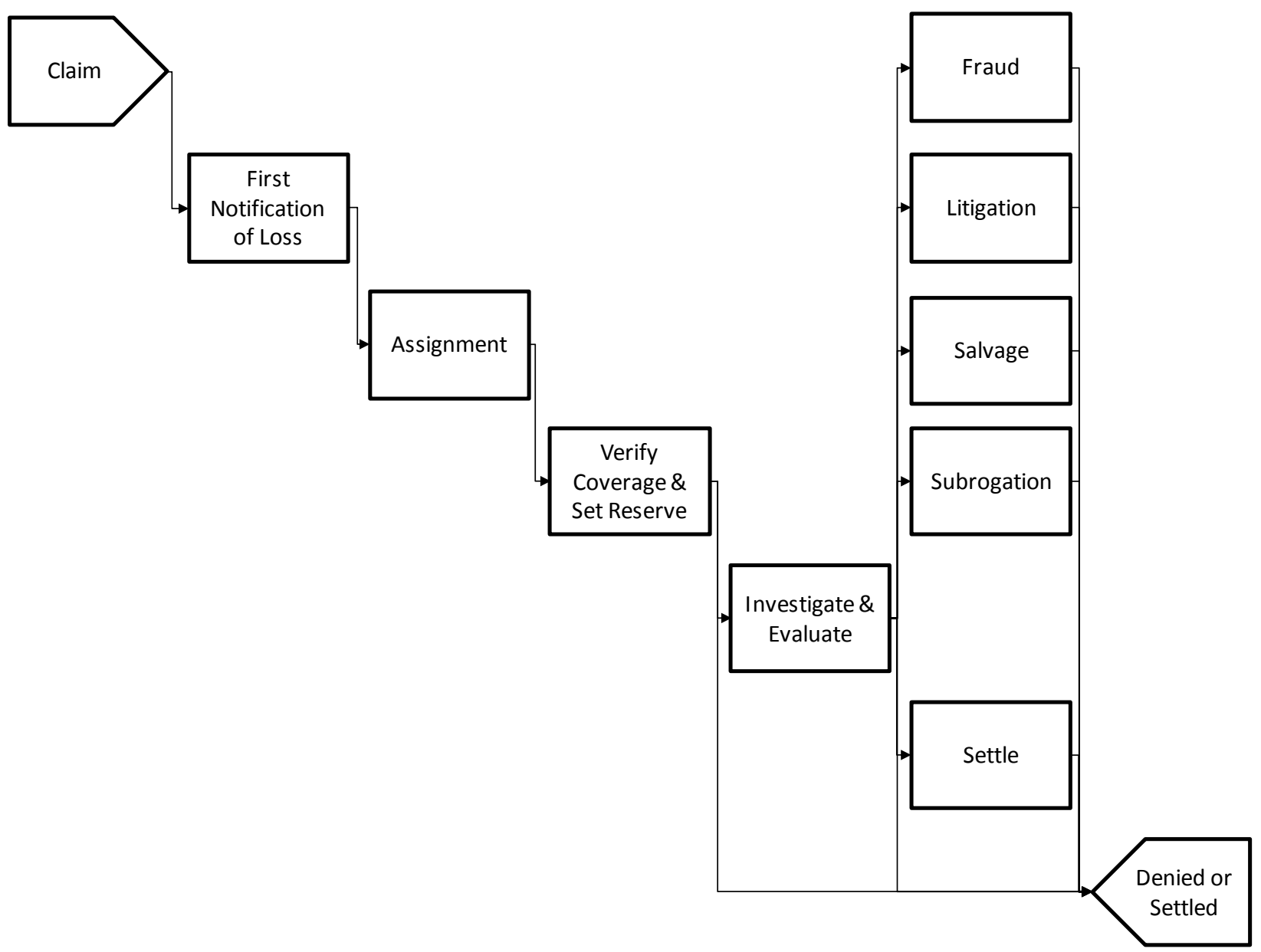

Figure 1: High-Level Claims Process Flow

\subsection{Assignment}

Once a claim is reported, it must be assigned to the appropriate adjuster. Adjusters often specialize in specific types of claims. Determining the appropriate adjuster to adjudicate a claim can be difficult given the limited amount of information available when a claim is first reported; because of this, as additional information is gathered, a claim is often reassigned to another adjuster.

\subsection{Verify Coverage and Set Initial Reserve}

Once the claim is assigned to the adjuster, his first task is to verify that the policy has coverage for the claim being reported.

\subsection{Investigation}

Investigation is the primary function of an adjuster. During this process the adjuster will interview relevant parties and gather appropriate documentation.

\subsection{Evaluate Investigation Results}

Now that all of the evidence has been compiled and the interviews completed, the adjuster is ready to evaluate the information. Different types of claims typically require indemnification of different types of 
costs. For example, many auto claims require having a rental car contracted because the claim involves the insured or claimant losing use of their vehicle while claims involving bodily injury or workers' compensation may incur medical treatment costs.

\subsection{Settlement and Payments}

Once the adjuster determines what is required to indemnify the claimant, payment is made. Settlement payments may be a single payment or a series of payments. For example, workers compensation claims for lost wages are often disbursed as recurring payments over months or years. In addition to paying parties involved in the claim, the adjuster must also make expense payments to cover other related expenses including car rental fees, prescriptions, outside investigators, and attorney fees.

During investigation and evaluation of the claim, the adjuster may also find that the evidence suggests denial of the claim reported. He is responsible for contacting the claimant regarding the denial.

\subsection{Fraud, Litigation, Salvage, and Subrogation}

Finally, the claim may need to be processed by other business units. If the adjuster suspects fraud based on suspicious findings during investigation the adjuster will refer the claim to the Special Investigations Unit (SIU) for a forensic investigation. Litigation could be required depending on the circumstances of the claim. In an auto or property damage claim, some parts of the vehicle or property may be salvageable and can be sold. Finally, the adjuster may determine that another insurance company or involved party may be responsible for some or all of the damages. For example, if a faulty drier part causes a fire in a home, the manufacturer of the drier would be responsible for covering the damages. In this case, the adjuster works with the insurer's subrogation unit to recover damages.

\section{THE SIMULATION MODEL}

In order to test process changes, automation, a new channel strategy and different resource levels, Westfield decided to build a simulation model using Rockwell Automation's Arena (Rockwell 2012). The first step in the model development was to map the process end-to-end, accounting for process steps, processing times, resources involved, business rules, and decision logic. Processing times were gathered from subject matter experts during the process mapping workshops where they were asked to provide the minimum, maximum and usual (mode) times it takes to complete each step. Once the process mapping workshops were completed, site visits and ride-alongs were conducted in order to validate the process maps as well as develop a deep understanding of the process.

Time-stamped data was extracted from Westfield's enterprise data warehouse and from the CCC logs to generate arrival patterns by channel. The current claims organizational structure was used to derive staffing levels for each resource type by office. In addition to the typical issues encountered with any simulation project such as non-stationary Poisson arrivals and a diverse resource pool, there were many unique features that the simulation model needed to mimic. These included 1) a never before seen queuing rule at the data entry vendor, 2) self-reported work hours for the allocation specialist, 3) re-seizing the same adjuster after information is returned to the adjuster, 4) accounting for the amount of time the adjuster spends driving to locations 5) using anomaly detection to determine when the CCC was in a storm situation versus business as usual, 6) processing the features that make up a claim, 7) using modified hybrid resources in the CCC, and 8) call abandonment in the CCC.

\subsection{Unique Queuing Rule}

Typically, work is queued in business processes according to one of three rules: First-In-First-Out (FIFO), Last-In-First-Out (LIFO), or ordered based on a value and select the item ranked first. However, management of the claims data entry had CSRs removing claims from the intake pile according to another rule. If there were more than four claims in the pile, the CSR was to take two from the top, one from the 
middle, and one from the bottom; otherwise take whatever is in the pile. Management was convinced that this shortened the average queuing time. However this queuing rule does not improve the average queuing time it only improves the average maximum time. Nonetheless, the approach was modeled in the simulation accordingly.

\subsection{Self-Report Working Hours}

Based on the site visits, it became obvious that allocation of claims to an adjuster was a potential bottleneck in the process since claims are manually assigned by an allocation specialist following receipt and input. If this process was not modeled correctly, there was a good chance the model would not be validated. The most important part was to understand the actual hours worked. In order to do that a macro was created to collect the timestamp of the email being sent from data entry, the timestamp of the email that was forwarded by the allocation specialist to the adjuster, and the claim key that was part of the subject line of the email. This data was used to validate the model from first notification of loss to assignment as well as determine the work hours for the allocation specialist.

Figure 2 displays the time of day the email was sent from the data entry vendor on the horizontal axis and the time that elapsed from that point until the email was sent to the adjuster once it was assigned.

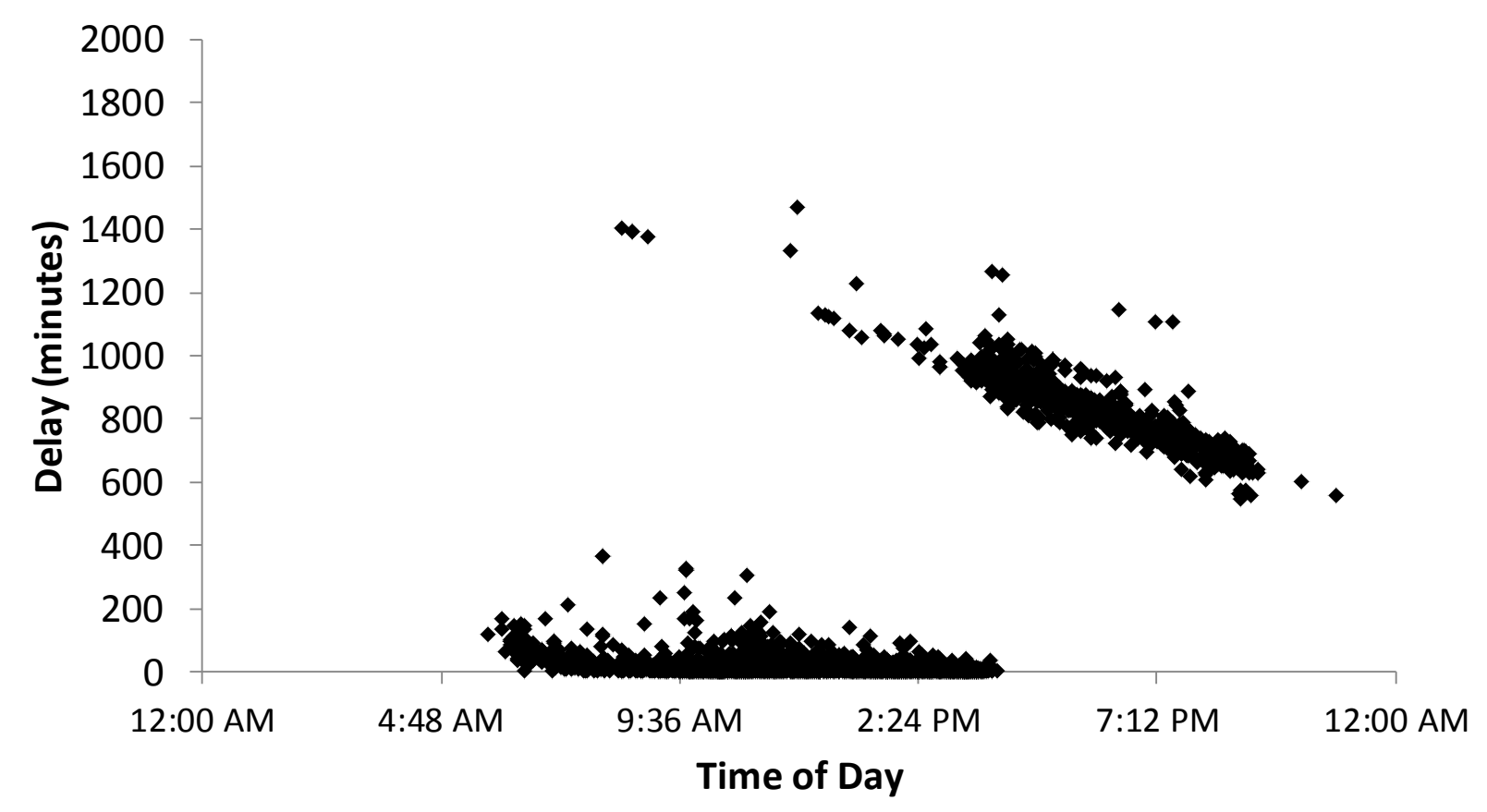

Figure 2: Allocation Specialist's Operating Hours

The chart shows two distinct groups of data: 1) the lower grouping contains claims that arrive when the allocation specialist is at the office and quickly assigns the claim, and 2) the upper grouping contains claims that arrive after the allocation specialist has left the office and the claim must wait until the specialist returns the next business day. The results from this analysis were used to accurately set the schedule for the allocation specialist resource in the model.

\subsection{Re-seizing the Same Adjuster}

Once a claim is assigned to an adjuster, the adjuster works the claim until it is settled or denied. In addition, each adjuster type within each of Westfield's many service offices has their own queue for work to 
be processed. Therefore, the model needed to output statistics for each office and seize the correct resource depending on the process step.

The need to release and re-seize a resource occurs when an adjuster must wait for additional documentation from an involved party or external vendor. When that happens the request is sent and then the adjuster waits to receive the documentation. Once the documentation is returned it must be queued in the correct queue for the correct resource. This requirement was modeled by storing the adjuster assignment and associated queue with each claim (entity) in the model using entity attributes. The claim then queues and seizes in the model based on those attributes.

\subsection{Adjuster Drive Time}

Another process phenomenon that was observed during site visits was the inordinate amount of time the adjuster spends driving to locations, affectionately referred to by adjusters as windshield time. This time could be as little as ten minutes to as much as several hours. In order to account for this time in the model, the claim county and the office county were determined for each claim over a one year period from Westfield's enterprise data warehouse. The average commute speeds for those counties, the latitude and longitude of the offices, the latitude and longitude of the accident counties, and the spherical law of cosines were used to create windshield time datasets. These datasets were then used to create windshield time distributions using ExpertFit.

\subsection{Determining Business As Usual}

While casualty and workers' compensation claims arrive consistently throughout the year, auto and property claims can be directly impacted by weather. Claims reported volume also fluctuates depending on the day of the week and time of year. IBM's SPSS Modeler was used for anomaly detection based on the number claims reported, claim type, month reported, and weekday reported. The results of this analysis identified claim arrivals that were anomalous, which were removed from average weekly arrival calculations. Figure 3 demonstrates property claim anomalies associated with extreme weather events.

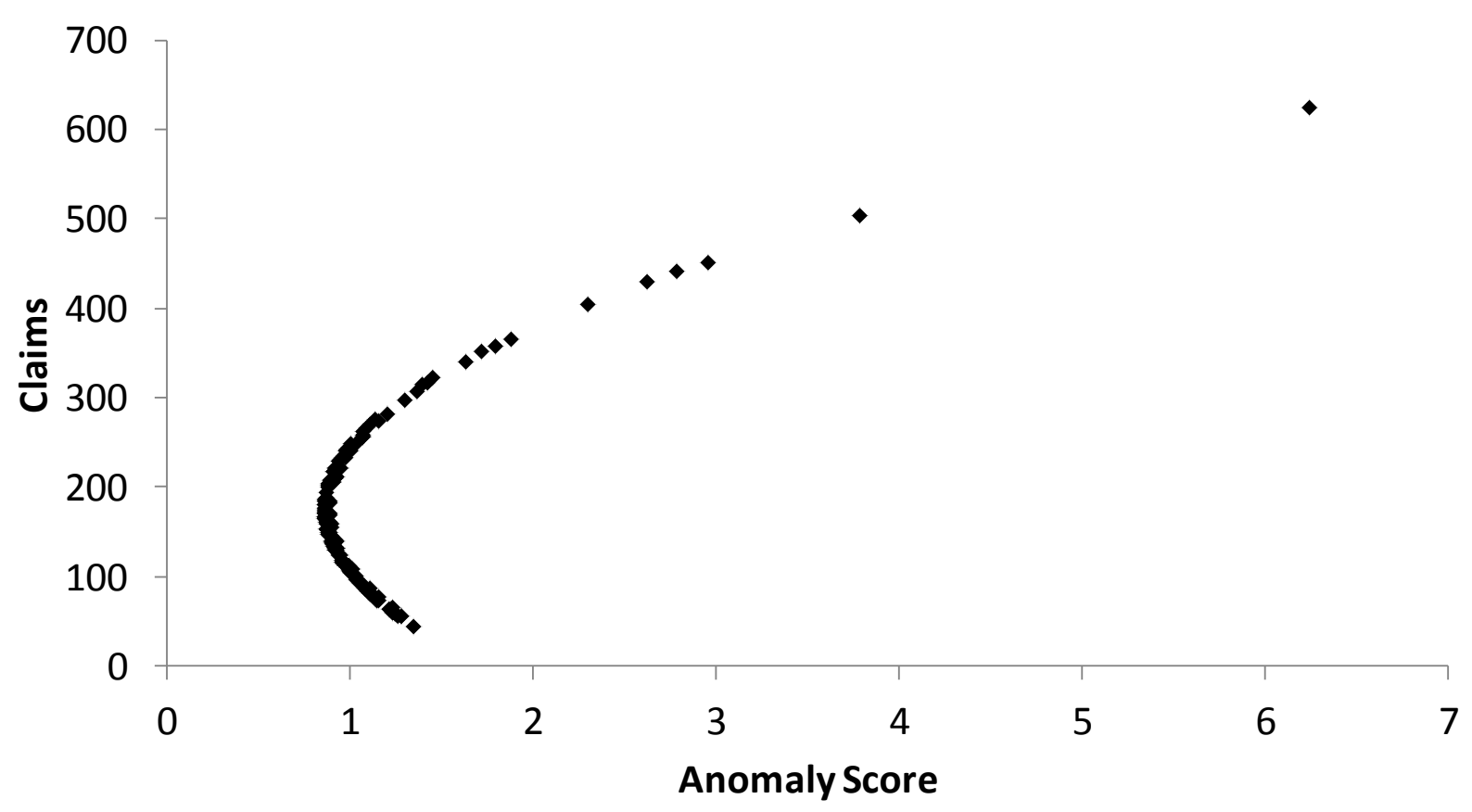

Figure 3: Anomaly Detection for Determining Business As Usual for Property Claims 
In order to validate the model, we needed to simulate periods of time that did not include significant anomalies such as storms. By removing storm weeks from the input and validation data, we were able to validate the model based on business as usual arrivals and workload, but then experiment with the impacts of storm activity on the process.

\subsection{Processing Claim Features}

Each claim is made up of one or more features. A feature is a coverage that applies to a specific party involved in the loss. For example, an auto accident involving a deer hit may have two features; comprehensive to repair the insured's vehicle, and rental reimbursement to pay for the insured's rental while the vehicle is being repaired. Therefore, all claims are not equal in terms of the amount of work they generate.

This was accounted for in the simulation through duplication based on historical data by claim type. The most interesting part of the challenge with processing features was grouping them back together to assign the cumulative claim reserve to each feature, and holding all features at the end of the simulation until they are able to be grouped together to close the claim.

Throughout validation efforts, the authors learned to use the COMBINE block instead of the GROUP block in Arena (Rockwell 2012) where appropriate. Using the combine block dramatically reduced the number of entities in the system, enabling faster processing and ultimately ending the array size and memory limitation problems encountered with the simulation software.

\subsection{Modified Hybrid Approach to Resources}

Westfield's CCC not only processes claims, it also handles the intake of billing calls. The CCC is split into two areas that process claims and billing separately, with some resources that process both. The resources that process both can be designated hybrid (primarily answer one call type and answer the opposite for calls that have waited longer than the service level) or hybrid (answer the next call, claims or billing). In order to model the claims processing accurately, the hybrid resources were also seized by arrivals to the billing channel. For an in-depth discussion of the modified approach to resources, see Conley and Grabau (in press).

\subsection{Call Abandonment}

Call abandonment in Westfield's CCC posed a modeling challenge. Westfield measures service level by dividing the number of calls answered under a certain number of seconds by the number of calls answered. Calls that abandon directly impact the service level by artificially raising it if the abandoned call waited longer than the target service time. Challenges with validating service level in the CCC necessitated a more accurate method for modeling abandonment. For further insight into this method, see Conley (in press).

\section{RESULTS}

The simulation model has been used to drive significant changes in the implementation of the claims management system (CMS). Initially the requirement for automated adjuster assignment was removed from the list of requirements the vendor was to implement in the CMS. The simulation experiments showed that implementing automated adjuster assignment would lead to the single largest reduction in the cycle time from first notification of loss to adjuster assignment. Automated assignment was then appropriately reprioritized.

As mentioned in section 3.2, the allocation specialist's hours represented a bottleneck in the process as claims arriving later in the day waited until the next business day to be assigned. Further analysis, displayed in Figure 4, showed the significant proportion of claims that arrived after 4 p.m. 


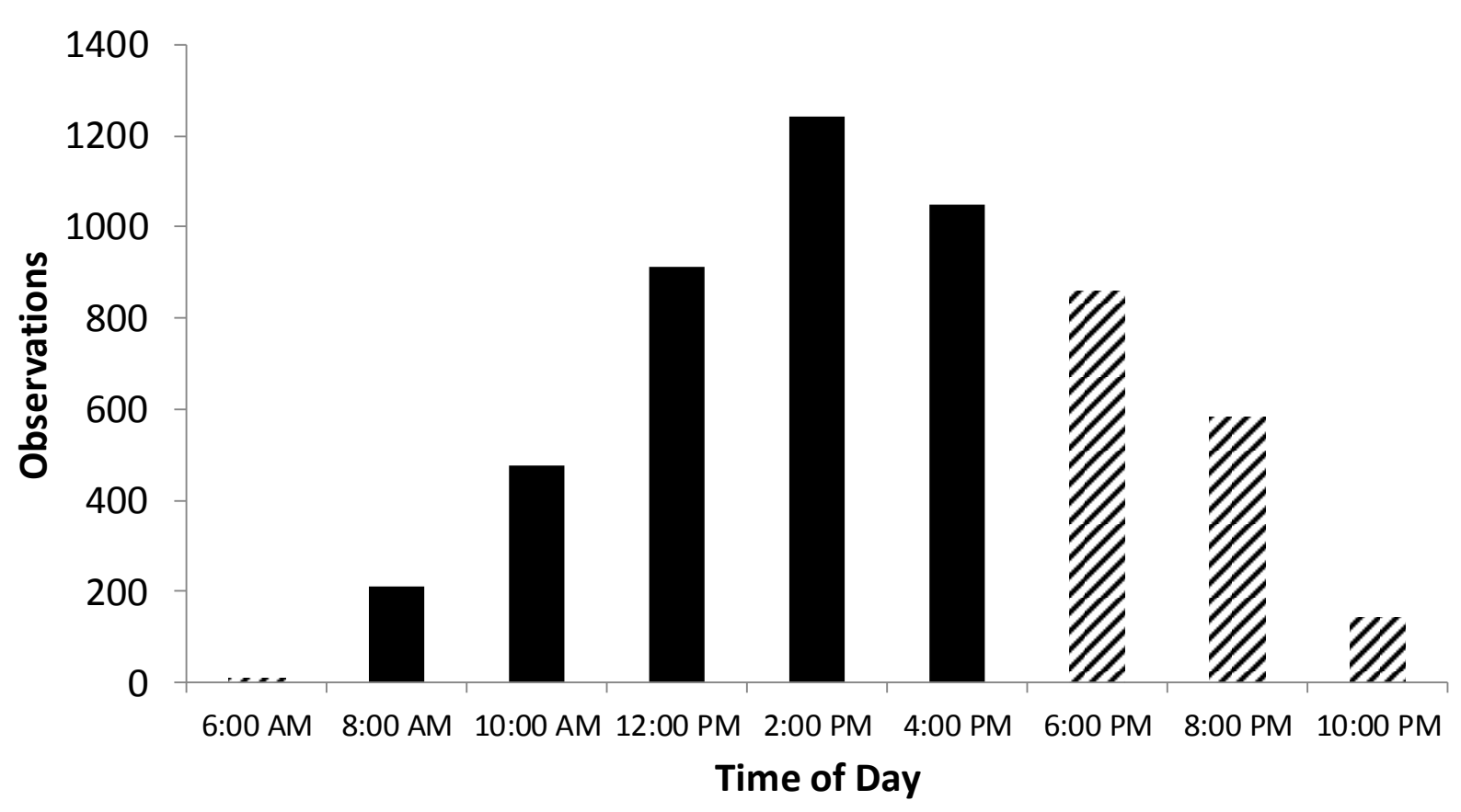

Figure 4: Claim Arrival Distribution at the Office by Time of Day

By 4 p.m. the allocation specialist had already left for the day or the weekend, so those claims were waiting overnight, at a minimum. This is why it was necessary to enter the allocation specialist's hours correctly, requiring claims that arrived after-hours to wait longer. If this had not been accounted for, the model would not have validated based on cycle time. An experiment was also conducted that demonstrated the value of having allocation specialists working back-to-back shifts in the office.

Prior to the simulation project, Westfield believed that there was an excessive delay between the data entry of a claim and the submission of the claim to the allocation specialist. The data analysis required to conduct the simulation indicated that the delay was 45 minutes on average and the vast majority of the email followed within one hour as depicted in Figure 5. 
Grabau, Conley, and Marshall

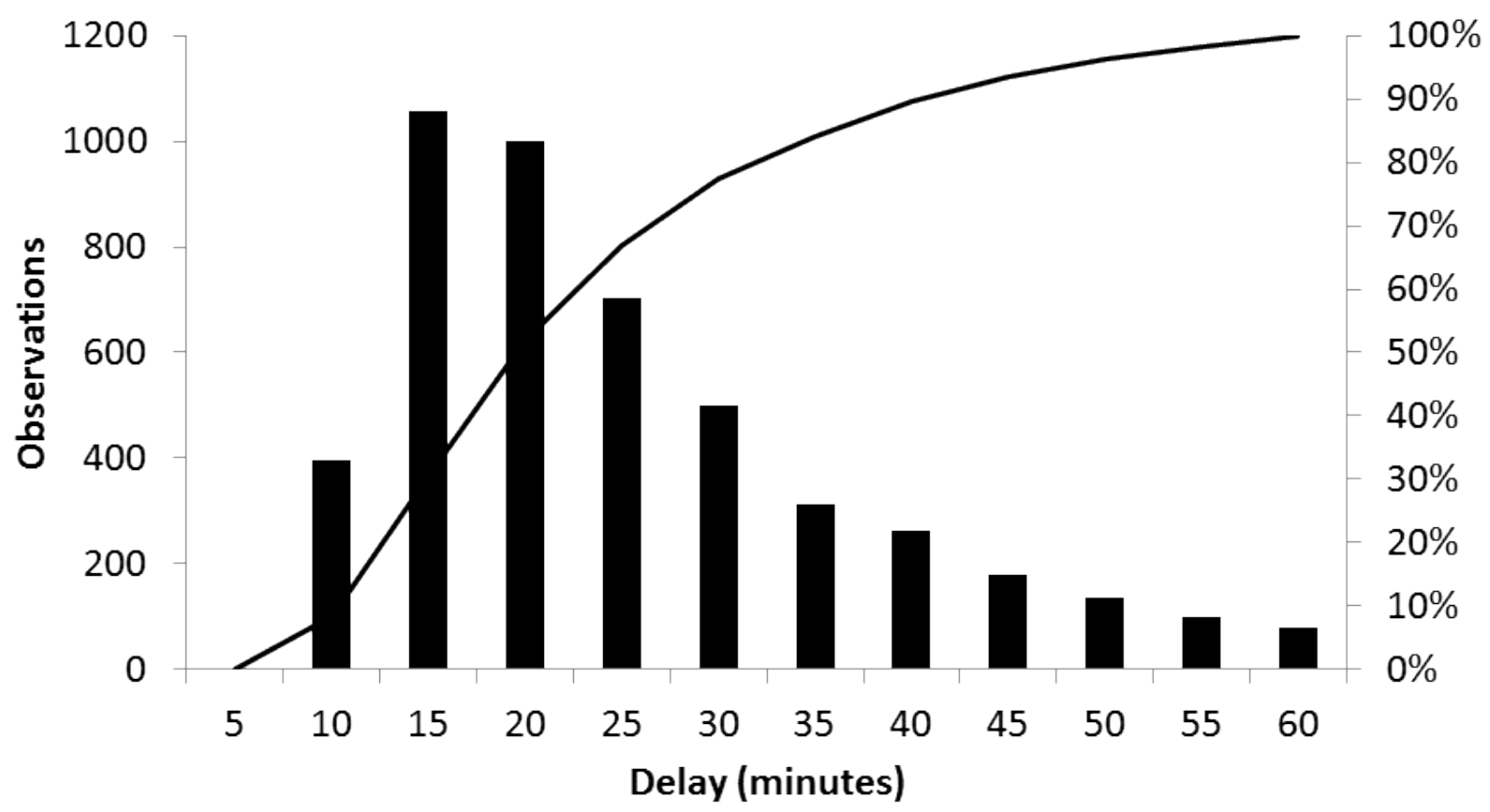

Figure 5: Delay from TTD to Email

This discovery diverted Westfield's attention away from data entry to other process changes where the impacts would be more profound.

\section{CONCLUSIONS}

Unlike management solutions with spreadsheets that are often based on averages, or static workflow diagrams, simulation captures the end-to-end interdependencies and variability among processes and resources. Instead of an implement, wait, and measure approach, simulation enables a system to be studied over time without actually impacting that system because months or even years of activity can be simulated in a matter of minutes or seconds. Simulation also allows different strategies to be studied in a lowcost, risk-free environment on a laptop prior to implementation, resulting in better requirements, better ideas, and more successful implementations.

The ability of a simulation to model the complexities and dependencies inherent in processing insurance claims and experiment with changes in people, processes, and technology prior to implementation is unparalleled. Westfield is applying this advanced analytics technique to provide insights and drive better decision making during the implementation of their CMS, enabling the organization to "go live" smartly the first time.

Buy-in from the executives was crucial and was obtained with the fact-based analyses the simulation provided as well as the realistic depiction of the process in the animation. The analysts at Westfield are now building a fifth simulation model that focuses on the specific business processes of the SIU.

\section{REFERENCES}

Conley, Q. D. In press. "Simulating Abandonment Using Kaplan-Meier Survival Analysis in a Shared Billing and Claims Call Center." In Proceedings of the 2013 Winter Simulation Conference, Edited by R. Pasupathy, S.-H. Kim, A. Tolk, R. Hill, and M. E. Kuhl. Piscataway, New Jersey: Institute of Electrical and Electronics Engineers, Inc. 
Conley, Q. D., M. R. Grabau. In press. "Simulating a Modified Hybrid Approach to Resource Assignment in a Shared Billing and Claims Call Center." In Proceedings of the 2013 Winter Simulation Conference, Edited by R. Pasupathy, S.-H. Kim, A. Tolk, R. Hill, and M. E. Kuhl. Piscataway, New Jersey: Institute of Electrical and Electronics Engineers, Inc.

Law, A. L. 2006. Simulation Modeling and Analysis. 4th ed. New York, New York: McGraw-Hill, Inc. Rockwell Automation, Inc. 2012. Arena (Version 14.0). Milwaukee, WI.

\section{AUTHOR BIOGRAPHIES}

MARK GRABAU is an Associate Partner in IBM's Business Analytics and Optimization service line. He leads the North America Advanced Analytics and Optimization practice for the Financial Services Sector at IBM and has over 20 years of experience building advance analytics models supporting the marketing and operational functions of large Financial Services, Retail, and Government organizations. Mr. Grabau has managed large, complex analytics projects while providing technical oversight to model design, development, and deployment. He has published 12 refereed papers on the application of advanced analytics to real-world business problems. Mr. Grabau is a Lean Six Sigma Blackbelt and holds M.S. degrees in Operations Research and Statistics from the Colorado School of Mines and a B.S. in Operations Research from the United States Air Force Academy. His email address is mark.grabau@us.ibm.com.

QUINN CONLEY is a Business Intelligence Analyst in the Analytics Resource Center at Westfield Insurance. He currently works as an internal, advanced-analytics consultant, focusing on discrete event simulation and predictive modeling. Prior to his current role, Mr. Conley provided analytics support in the rate and profitability management of Westfield's non-standard auto products. Mr. Conley is a graduate of Baldwin-Wallace College in Berea, Ohio; with a B.S. in Mathematics. He will be graduating from Ashland University in Ashland, Ohio in Fall, 2013 with a Master's in Business Administration. His email address is quinnconley@westfieldgrp.com.

MELISSA MARSHALL is a Business Intelligence Analyst within the Analytics Resource Center. For the last 3 years, Ms. Marshall has worked on the development and implementation of Business Intelligence across the organization, and is now delving into more advanced analytic projects such as process simulation. Prior to her current role, she worked as an Associate Financial Analyst within the Personal Lines Product Department, supporting Personal Lines product development. Ms. Marshall is a graduate of Youngstown State University with a BS degree in Mathematics. She also holds an MS degree in Statistics from The University of Akron and is currently a part-time faculty member in their Department of Statistics. Her email address is melissamarshall@westfieldgrp.com. 